MIT-CTP-4209

\title{
Localizing fields on brane in magnetized backgound
}

\author{
Shih-Hao Hda \\ Center for Theoretical Physics, Massachusetts Institute of Technology, \\ Cambridge, Massachusetts 02139, USA \\ C. Q. Gengb \\ Department of Physics, National Tsing Hua University, Hsinchu, Taiwan 300 \\ National Center for Theoretical Sciences, Hsinchu, Taiwan 300
}

\begin{abstract}
To localize the scalar, fermion, and abelian gauge fields on our 3-brane, a simple mechanism with a hypothetical "magnetic field" in the bulk is proposed. This mechanism is to treat all fields in the equal footing without ad hoc consideration. In addition, the machanism can be easily realized in a flat dimension six Minkowski space and it works even in the weak coupling limit.
\end{abstract}

\footnotetext{
a E-mail address: shho@mit.edu

b E-mail address: geng@phys.nthu.edu.tw
} 
It is known that the brane-world scenario allows us to use infinite extra dimensions without compactification. Since the low energy effective theory should be four-dimensional, the mechanism to localize matter fields naturally on a brane still is a main topic in the brane-world scenario [1]. In Ref. [2], Rubakov and Shaposhinikov proposed to localize fields on our 3-brane by topological defect:1 The mechanism to localize scalar and fermion is essentially given in Refs. [4, 5]. The feature of the mechanism is the existence of zero modes, identified to be our standard model fields. In the literature, there have been many proposals to trap fields in our four-dimesional world [1]. In this paper, we try to illustrate a simple and old way to localize particles on a plane by applying a strong magnetic field.

Let us consider the Lagrangian in a theory with a massive complex scalar field $\phi$, and a (classical) background gauge field $a_{M}$ associated with a $U(1)_{X}$ in a flat dimension six spacetime 2

$$
\mathcal{L}_{S}=\left(\partial_{M}+i g a_{M}\right) \phi^{*}\left(\partial^{M}-i g a^{M}\right) \phi-m_{s}^{2} \phi^{*} \phi
$$

The equation of motion for these fields can be easily derived to be

$$
\left[\partial_{M} \partial^{M}-i g\left(\partial_{M} a^{M}\right)-2 i g a_{M} \partial^{M}-g^{2} a_{M} a^{M}+m_{s}^{2}\right] \phi=0 .
$$

For the case in which "magnetic field" is present along the extra dimensions, i.e. $f_{45}=$ $-f_{54} \equiv B, f_{\mu \nu}=0$ for $\mu, \nu=0,1,2$ and 3 in the gauge $a_{M}=\left(0,0,0,0,-B x_{5} / 2, B x_{4} / 2\right)$ with $f_{M N} \equiv \partial_{M} a_{N}-\partial_{N} a_{M} \cdot 3$ Eq.(2) becomes 4

$$
\left[\eta^{\mu \nu} \partial_{\mu} \partial_{\nu}+\left(i \partial_{4}-\frac{g B}{2} x_{5}\right)^{2}+\left(i \partial_{5}+\frac{g B}{2} x_{4}\right)^{2}+m_{s}^{2}\right] \phi=0 .
$$

Clearly, it reduces to a non-relativistic quantum mechanics problem. Using the raising and lowering operator method, we obtain the energy spectrum and ground state wave function:

$$
\begin{aligned}
& p_{0}^{2}=p_{1}^{2}+p_{2}^{2}+p_{3}^{2}+(2 n+1)|g B|+m_{s}^{2}, n=0,1,2, \cdots \\
& \phi_{n=0}=N_{s} e^{i p_{\mu} x^{\mu}} r^{l} e^{i l \theta} e^{-|g B| r^{2} / 4}, l=0,1,2, \cdots
\end{aligned}
$$

${ }^{1}$ In Ref. [3], the author attempted to reproduce SM phenomenology exploiting the type of fermion localization by the mechanism introduced in Ref. [2]

${ }^{2}$ Our convention is $\eta^{M N}=\operatorname{diag}(+1,-1,-1,-1,-1,-1)$, while Greek and Latin indices represent the $(3+1)$ spacetime and all the dimensions indices, respectively.

3 The wavefunctions depend on the gauge choice and they differ only by a phase factor as expected [6].

${ }^{4}$ Here we note the location of this 3-brane along the extra dimensions is arbitrary and it also depends on the gauge choice of classical field $a_{M}$. For instance, the choice of $a_{M}(x)=\left(0,0,0,0,-B\left(x_{5}+a\right) / 2, B\left(x_{4}+b\right) / 2\right)$ with some constants $a, b$ will give us the same background "magnetic field" but it will also change the location of the brane from $x_{4}=x_{5}=0$ to $x_{4}=-b, x_{5}=-a$. But the important property is that the wavefunctions and the propagators fall off exponentially outside the brane. 
representing the Landau levels for spin-zero particles with the lowest Landau level (LLL) for $\mathrm{n}=0$ of the ground state energy $E_{0}^{2}=p_{1}^{2}+p_{2}^{2}+p_{3}^{2}+|g B|+m_{s}^{2}$, where $N_{s}$ is the normalization constant, $r^{2}=x_{4}^{2}+x_{5}^{2}$ and $\tan \theta=x_{5} / x_{4}$. This shows that the ground state with respect to the bulk dynamics is localized near $r^{2} \equiv r_{0}^{2}=2 l /|g B|$ as long as the bulk magnetic field is large enough. However, we observe that, in the presence of the term $|g B|$, these "zero modes" 5 carry information from extra dimensions even if the bulk mass is absent.

We emphasis that the "center" of wavefunction in Eq.(5) is $r=r_{0}$, which depends on the angular quantum number $l$. Hence the field is concentrated at $r=r_{0}(l)$ with characteristic length $1 /|g B|$ for a fixed 6 , we cannot argue which value of $l$ is a preferred one since all values of $l$ correspond to the LLL. The absolute position in extra dimensions, however, is irrelevant in our consideration, the relevant quantity is always the relative positions of different fields along the extra dimensions. We believe this will become much clearer when the interactions between fields are taken into account. For present purpose, we emphasis the dynamics of fields is effectively confined on 3-brane even if we consider the free fields in an magnetized background. This can be seen as we consider the Green's function in the second part of this paper.

We now turn to the fermion case, the goal is still to find a zero mode localized on our brane [7]. The Lagrangian for a massive Dirac fermion $\Psi$ on a background gauge field $a_{M}$ in a flat dimension six spacetime is

$$
\mathcal{L}_{D}=\bar{\Psi}\left(i D_{M} \Gamma^{M}-m_{f}\right) \Psi
$$

where $D_{M} \equiv \partial_{M}-i g a_{M}$ with the coupling constant $g$ and the representations of 8 by 8 Dirac matrices are chosen to be:

$$
\begin{aligned}
& \Gamma^{\mu}=\gamma^{\mu} \otimes \sigma_{1}=\left(\begin{array}{cc}
0 & \gamma^{\mu} \\
\gamma^{\mu} & 0
\end{array}\right), \Gamma^{4}=i \gamma^{5} \otimes \sigma_{1}=\left(\begin{array}{cc}
0 & i \gamma^{5} \\
i \gamma^{5} & 0
\end{array}\right), \\
& \Gamma^{5}=1 \otimes i \sigma_{2}=\left(\begin{array}{cc}
0 & 1 \\
-1 & 0
\end{array}\right), \Gamma^{7}=\Gamma^{0} \Gamma^{1} \Gamma^{2} \Gamma^{3} \Gamma^{4} \Gamma^{5}=\left(\begin{array}{cc}
1 & 0 \\
0 & -1
\end{array}\right),
\end{aligned}
$$

with the four dimensional gamma matrices in the chiral representation. The gamma matrices

\footnotetext{
5 The "zero mode" referred by us means "zero mode of Landau level", i.e. the Lowest Landau Level (LLL).

${ }^{6}$ This can be seen more clearly in Landau gauge.
} 
in Eq. (7) satisfy the Clifford algebra

$$
\begin{aligned}
\left\{\Gamma^{M}, \Gamma^{N}\right\} & =2 \eta^{M N}, \eta^{M N}=\operatorname{diag}(+1,-1,-1,-1,-1,-1), \\
\left\{\Gamma^{M}, \Gamma^{7}\right\} & =0,\left(\Gamma^{7}\right)^{2}=1,
\end{aligned}
$$

with $\mathrm{M}, \mathrm{N}=0,1,2,3,4,5$. By defining the $6 \mathrm{D}$ chiral states for the eight-componet spinor $\Psi$ :

$$
\Psi=\left(\begin{array}{c}
\Psi_{+} \\
\Psi_{-}
\end{array}\right) \text {, with } \Gamma^{7} \Psi_{ \pm}= \pm \Psi_{ \pm},
$$

we can decompose equations of motion for $\Psi_{ \pm}$into

$$
\begin{aligned}
& \left(i \gamma^{\mu} D_{\mu}-\gamma^{5} D_{4}+i 1 D_{5}\right) \Psi_{-}=m_{f} \Psi_{+} \\
& \left(i \gamma^{\mu} D_{\mu}-\gamma^{5} D_{4}-i 1 D_{5}\right) \Psi_{+}=m_{f} \Psi_{-}
\end{aligned}
$$

Consequently, these equations can be rewritten as

$$
\begin{aligned}
& \left(i \gamma^{\mu} D_{\mu}-\gamma^{5} D_{4}-i 1 D_{5}\right)\left(i \gamma^{\mu} D_{\mu}-\gamma^{5} D_{4}+i 1 D_{5}\right) \Psi_{-}=m_{f}^{2} \Psi_{-}, \\
& \left(i \gamma^{\mu} D_{\mu}-\gamma^{5} D_{4}+i 1 D_{5}\right)\left(i \gamma^{\mu} D_{\mu}-\gamma^{5} D_{4}-i 1 D_{5}\right) \Psi_{+}=m_{f}^{2} \Psi_{+},
\end{aligned}
$$

which lead to

$$
\begin{aligned}
& {\left[\eta^{\mu \nu} \partial_{\mu} \partial_{\nu}+\left(i \partial_{4}-\frac{g B}{2} x_{5}\right)^{2}+\left(i \partial_{5}+\frac{g B}{2} x_{4}\right)^{2}+g B \gamma^{5}+m_{f}^{2}\right] \Psi_{-}=0} \\
& {\left[\eta^{\mu \nu} \partial_{\mu} \partial_{\nu}+\left(i \partial_{4}-\frac{g B}{2} x_{5}\right)^{2}+\left(i \partial_{5}+\frac{g B}{2} x_{4}\right)^{2}-g B \gamma^{5}+m_{f}^{2}\right] \Psi_{+}=0}
\end{aligned}
$$

Since $\gamma^{5}$ commutes with the whole operator in the brackets in Eqs. (15) and (16), it can be replaced by the eigenvalue $\gamma$ which is the chirality of a $4 \mathrm{D}$ fermion:

$$
\Psi_{ \pm}=\left(\begin{array}{c}
\Psi_{ \pm_{L}} \\
\Psi_{ \pm_{R}}
\end{array}\right), \gamma^{5} \Psi_{ \pm_{L, R}}=\gamma \Psi_{ \pm_{L, R}},
$$

with $\gamma=-1(+1)$ for $\mathrm{L}(\mathrm{R})$. We see that both Eqs. (15) and (16) take the same form as Eq. (3). Therefore, we can write down the energy spectrum and ground state wavefunction immediately:

$$
\begin{aligned}
& p_{0}^{2}=p_{1}^{2}+p_{2}^{2}+p_{3}^{2}+(2 n+1+\operatorname{sgn}(g B) \gamma)|g B|+m_{f}^{2}, n=0,1,2, \cdots \text { for } \Psi_{-} \\
& p_{0}^{2}=p_{1}^{2}+p_{2}^{2}+p_{3}^{2}+(2 n+1-\operatorname{sgn}(g B) \gamma)|g B|+m_{f}^{2}, n=0,1,2, \cdots \text { for } \Psi_{+} \\
& \Psi_{ \pm_{n=0}}=N_{f} e^{i p_{\mu} x^{\mu}} r^{l} e^{i l \theta} e^{-|g B| r^{2} / 4} \omega_{\gamma}, l=0,1,2,3 \ldots
\end{aligned}
$$


where $N_{f}$ is the normalization constant, $\omega_{\gamma}$ denotes the eigenvector of $\gamma^{5}, r^{2}=x_{4}^{2}+x_{5}^{2}$ and $\tan \theta=x_{5} / x_{4}$ as mentioned early. Now, we observe that for the lowest energy state with $n=0$ and $\gamma= \pm \operatorname{sgn}(g B)$ for $\Psi_{ \pm}$, the energy is $E_{0}^{2}=p_{1}^{2}+p_{2}^{2}+p_{3}^{2}$ if the bulk mass is absent. Clearly, there exist two zero modes with respect to the bulk dynamics, corresponding to $\Psi_{-L}$ and $\Psi_{+R}$ if $\operatorname{sgn}(g B)>0$.

Next, we consider the simplest case of complex massive vector gauge fields $A_{M}$ associated with $U(1)_{A}$, which also carry a charge of $U(1)_{X}$, i.e. $A_{M} \rightarrow A_{M}+\partial_{M} \Lambda(x)$ and $A_{M} \rightarrow$ $e^{i \alpha(x)} A_{M}$ under $U(1)_{A}$ and $U(1)_{X}$ gauge transformations, respectively. Based on Ref. [8], we can construct a gauge invariant Lagrangian under these two $U(1)$ groups with the help of a scalar Stueckelberg field $\rho$ whose transformation laws are $\rho \rightarrow \rho+m_{v} \Lambda^{\prime}(x)$ and $\rho \rightarrow e^{i \alpha(x)} \rho$ under $U(1)_{A}$ and $U(1)_{X}$, respectively [9]. Then, the Langrangian reads:

$$
\mathcal{L}_{V}=\frac{-1}{2} \mathbb{F}_{M N}^{*} \mathbb{F}^{M N}-i g f^{N M} V_{N}^{*} V_{M}+m_{v}^{2} V_{M}^{*} V^{M}-\frac{1}{\xi}\left|D_{M} A^{M}+\xi m_{v} \rho\right|^{2},
$$

where $V_{M} \equiv A_{M}-\frac{1}{m} D_{M} \rho, \mathbb{F}_{M N} \equiv D_{M} A_{N}-D_{N} A_{M}$ and $\xi$ is the gauge parameter. The gauge invariance of $U(1)_{X}$ is obvious and the invariance under $U(1)_{A}$ is guaranteed by the nontrivial condition of $D_{M} \Lambda^{\prime}=\partial_{M} \Lambda .7$ We can derive equations of motion by varying $A_{M}$ and $\rho$, given by

$$
\begin{gathered}
\left(D^{2} \eta^{M N}-D^{M} D^{N}\right) A_{M}+D_{M}\left(\frac{i g}{m_{v}} f^{M N} \rho\right)-i f^{N M} A_{M}+\frac{i g}{m_{v}} f^{N M} D_{M} \rho \\
+m_{v}^{2}\left(A^{N}-\frac{1}{m_{v}} D^{N} \rho\right)+\frac{1}{\xi}\left(D^{N} D^{M} A_{M}+m_{v} D^{N} \rho\right)=0
\end{gathered}
$$

and

$$
D_{N}\left(\frac{1}{m_{v}} D_{M} \mathbb{F}^{M N}-\frac{i g}{m_{v}} f^{N M}\left(A_{M}-\frac{1}{m_{v}} D_{M} \rho\right)+m_{v}\left(A^{N}-\frac{1}{m_{v}} D^{N} \rho\right)\right)-m_{v}^{2} \rho=0 .
$$

We will concentrate on the case with $f^{M N}=$ constant and choose the Feynman gauge $\xi=1$. Consequently, Eq.(22) is reduced td 8

$$
\left[\left(D^{2}+m_{v}^{2}\right) \eta^{M N}-2 i g f^{M N}\right] A(x) \epsilon_{M}=0 .
$$

${ }^{7}$ Note that $\left(D_{M} D^{M}+\xi m^{2}\right) \Lambda^{\prime}=0$.

${ }^{8}$ In the original Stuckelberg formalism, the vector field $A_{M}$ and scalar field $\rho$ satisfy the same field equation [9]:

$$
\begin{aligned}
& \left(\partial_{M} \partial^{M}+m^{2}\right) A_{M}=0 \\
& \left(\partial_{M} \partial^{M}+m^{2}\right) \rho=0
\end{aligned}
$$

In our consideration, the field equations are not the same owing to an external background field. We can derive the equation of motion for scalar field $\rho$ from Eq. (23) by using the relation $\left[D_{M}, D_{N}\right]=-i g f_{M N}$ :

$$
\left(D_{M} D^{M} 5+m^{2}\right) \rho=0 .
$$


Here, we still have degree of freedoms to set $A_{0}=0$ and write $A_{M} \equiv A(x) \epsilon_{M}$ with the polarization vector $\epsilon_{M}$

$$
\epsilon_{\mu}=\left(\begin{array}{c}
\delta_{\mu 1} \\
\delta_{\mu 2} \\
\delta_{\mu 3} \\
0 \\
0
\end{array}\right), \mu=1,2,3 ; \epsilon_{ \pm}=\frac{1}{\sqrt{2}}\left(\begin{array}{c}
0 \\
0 \\
0 \\
1 \\
\pm i
\end{array}\right) .
$$

In this basis, Eq. (25) becomes diagonal, given by

$$
\left[\left(D^{2}+m_{v}^{2}\right) \eta^{M N}+2 g B \chi g^{M N}\right] A(x) \epsilon_{M}=0,
$$

where $\chi=0$ for $M=1,2,3$ and $\chi=\mp 1$ for $M= \pm$. Hence, the energy spectrum and coordinate space component of $A_{M}$ is the solution of Eq.(3) with the replacement $m^{2} \rightarrow m^{2}+2 g B \chi$, found to be

$$
\begin{aligned}
& p_{0}^{2}=p_{1}^{2}+p_{2}^{2}+p_{3}^{2}+(2 n+1+2 \operatorname{sgn}(g B) \chi)|g B|+m_{v}^{2}, n=0,1,2, \cdots \\
& A(x)_{n=0}=N_{v} e^{i p_{\mu} x^{\mu}} r^{l} e^{i l \theta} e^{-|g B| r^{2} / 4}, l=0,1,2, \cdots
\end{aligned}
$$

where $N_{v}$ is the normalization constant, $r^{2}=x_{4}^{2}+x_{5}^{2}$ and $\tan \theta=x_{5} / x_{4}$. The ground state energy is $E_{0}^{2}=p_{1}^{2}+p_{2}^{2}+p_{3}^{2}+(1+2 \operatorname{sgn}(g B) \chi)|g B|+m_{v}^{2}$. Therefore, in the viewpoint of $4 \mathrm{D}$, we have a localized massive vector field $A_{\mu}$ with an effective mass $\sqrt{|g B|}$ in $4 \mathrm{D}$ and two localized scalar field $A_{ \pm}$which carry an extra degree of freedom with respect to the extra dimensional "handness" with effective squared masses of $m_{v}^{2}+(1+2 \operatorname{sgn}(g B))|g B|$ and $m_{v}^{2}+(1-2 \operatorname{sgn}(g B))|g B|$ for "right-handed" and "left-handed" states, respectively.

There are three possible cases of $A_{ \pm}$, which are listed in Table I. We note that in the third case, there is a heavy scalar for $\chi=-\operatorname{sgn}(g B)$ and a unstable mode for $\chi=\operatorname{sgn}(g B)$. This is the so-called zero mode problem and a general feature when we consider the spectrum of a vector field in the presence of magnetic field $[10,11] .9$ We also note that as a special case, to have a massless vector field $A_{\mu}$ in $4 \mathrm{D}$, we may re-consider the Lagrangian in Eq. (21) with an "imaginary" mass term as

$$
\mathcal{L}_{V}=\frac{-1}{2} \mathbb{F}_{M N}^{*} \mathbb{F}^{M N}-i g f^{N M} V_{N}^{*} V_{M}-\frac{1}{\xi}\left|D_{M} A^{M}+m_{v} \rho\right|^{2}+\left(i m_{v}^{\prime}\right)^{2} V_{M}^{*} V^{M},
$$

\footnotetext{
${ }^{9}$ In Ref. [11], a gauge theory with $\mathrm{U}(2)$ on a $T^{2}$ has been considered. There exists an instability in this guage theory, which corresponds to the instability in a brane-anti-brane configuration.
} 
TABLE I. Types of zero modes for $A_{ \pm}$

\begin{tabular}{|c|c|c|}
\hline Mass parameter & Effective masses of zero modes & Type of modes \\
\hline$m_{v}^{2}>|g B|$ & $\left.m_{e f f}^{2}\right|_{\chi= \pm 1}=m_{v}^{2}+(1 \mp 2 \operatorname{sgn}(g B))|g B|>0$ & $A_{ \pm}$are both massive \\
\hline$m_{v}^{2}=|g B|, \operatorname{sgn}(g B)>0$ & $\left.m_{e f f}^{2}\right|_{\chi=+1}=4|g B|,\left.m_{e f f}^{2}\right|_{\chi=-1}=0$ & $A_{+}$massless, $A_{-}$massive \\
$m_{v}^{2}=|g B|, \operatorname{sgn}(g B)<0$ & $\left.m_{e f f}^{2}\right|_{\chi=-1}=4|g B|,\left.m_{e f f}^{2}\right|_{\chi=+1}=0$ & $A_{-}$massless, $A_{+}$massive \\
\hline$m_{v}^{2}<|g B|, \operatorname{sgn}(g B)>0$ & $\left.m_{e f f}^{2}\right|_{\chi=+1}>0,\left.m_{e f f}^{2}\right|_{\chi=-1}<0$ & $A_{+}$tachyonic mode, $A_{-}$massive \\
$m_{v}^{2}<|g B|, \operatorname{sgn}(g B)<0$ & $\left.m_{e f f}^{2}\right|_{\chi=-1}>0,\left.m_{e f f}^{2}\right|_{\chi=+1}<0$ & $A_{-}$tachyonic mode, $A_{+}$massive \\
\hline
\end{tabular}

where $\left(m_{v}^{\prime}\right)^{2}=|g B|$.

Despite of the localized wavefunctions, we emphasize that the effective dynamics is confined on the 3-brane when the strong background field (and massless limit) is applied. This effective dimensional reduction can be seen in either the coordinate/momentum space reduction [12] or the phase space reduction [13]. The phase space reduction is examined in detail in Ref. [13]. Here, we would like to see the propagators for scalar and fermion fields and show that their propagations are indeed localized on the brane with the characteristic length $l_{B}^{-2} \equiv|g B|$.

Using Schwinger's proper time method [14], we can calculate the propagators of scalar and fermion fields in the presence of a constant background. Since the presence of the constant background field breaks the translational invariance, we recover the translation invariance by factoring out the Schwinger phase:

$$
\begin{aligned}
& G_{s}(x, y)=e^{i g \int_{y}^{x} a_{M}\left(\xi_{M}\right) d \xi^{M}} \tilde{G}_{s}(x-y), \\
& G_{f}(x, y)=e^{i g \int_{y}^{x} a_{M}\left(\xi_{M}\right) d \xi^{M}} \tilde{G}_{f}(x-y),
\end{aligned}
$$

where $G_{s}$ and $G_{f}$ stand for the propagators for scalars and fermions, respectively, and the path of the integral is a straight line.

The translational parts of the propagators $\tilde{G}_{s}$ and $\tilde{G}_{f}$ in the momentum space are given 
by

$$
\begin{aligned}
\tilde{G}_{s}(p)= & \int d^{6} x \tilde{G}_{s}(x) e^{i p x}=\int_{0}^{\infty} d s e^{-i s\left(m^{2}+p_{\mu}^{2}+\frac{\tan (|g B| s)}{|g B| s}\left(p_{4}^{2}+p_{5}^{2}\right)\right)} \frac{1}{\cos (|g B| s)}, \\
\tilde{G}_{f}(p)= & \int d^{6} x \tilde{G}_{f}(x) d^{i p x}=\int_{0}^{\infty} d s e^{-i s\left(m^{2}+p_{\mu}^{2}+\frac{\tan (|g B| s)}{|g B| s}\left(p_{4}^{2}+p_{5}^{2}\right)\right)} \times \\
& {\left[\left(p_{\mu} \Gamma^{\mu}+m\right)\left(1+\operatorname{sgn}(g B) \Gamma^{4} \Gamma^{5} \tan (|g B| s)\right)-\left(p_{4} \Gamma^{4}+p_{5} \Gamma^{5}\right)\left(1+\tan ^{2}(|g B| s)\right],\right.}
\end{aligned}
$$

which can be decomposed to be [12, 15]:

$$
\begin{aligned}
& \tilde{G}_{s}(p)=i e^{-\frac{p_{\perp}^{2}}{\mid g B} \mid} \sum_{n=0}^{\infty}(-1)^{n} \frac{L_{n}(x)}{p_{\mu}^{2}-m^{2}-(2 n+1)|g B|} \\
& \tilde{G}_{f}(p)=i e^{-\frac{p_{\perp}^{2}}{|g B|}} \sum_{n=0}^{\infty} \frac{(-1)^{n} D_{n}(g B, p)}{p_{\mu}^{2}-2|g B| n-m^{2}}
\end{aligned}
$$

respectively, with

$$
\begin{aligned}
D_{n}(g B, p)= & \left(p_{\mu} \Gamma^{\mu}+m\right)\left[\left(1+i \Gamma^{4} \Gamma^{5}\right) L_{n}^{0}(x)-\left(1+i \Gamma^{4} \Gamma^{5}\right) L_{n-1}^{0}(x)\right] \\
& +4\left(p_{4} \Gamma^{4}+p_{5} \Gamma^{5}\right) L_{n+1}^{1}(x)
\end{aligned}
$$

where $p_{\perp}^{2} \equiv p_{4}^{2}+p_{5}^{2}, x \equiv \frac{2\left(p_{4}^{2}+p_{5}^{2}\right)}{|g B|}$ and $L_{n}^{(\alpha)}(x)$ are the (associated) Laguerre polynomials. In the LLL for $n=0$, the propagators take the forms:

$$
\begin{aligned}
& \tilde{G}_{s}(p)=i e^{-\frac{p_{\perp}^{2}}{|g B|}} \frac{1}{p_{\mu}^{2}-m^{2}-|g B|} \\
& \tilde{G}_{f}(p)=i e^{-\frac{p_{\perp}^{2}}{|g B|} \frac{p_{\mu} \Gamma^{\mu}+m}{p_{\mu}^{2}-m^{2}}\left(1-i \Gamma_{4} \Gamma_{5}\right),}
\end{aligned}
$$

which are the exact free propagators in $(3+1)$ dimension in the region of $p_{\perp}^{2}<<|g B|$. We can also transform them back to the coordinate space:

$$
\begin{aligned}
& \tilde{G}_{s}(x-y)=\frac{1}{4 \pi l_{B}^{2}} e^{-\frac{\left(x_{4}-y_{4}\right)^{2}+\left(x_{5}-y_{5}\right)^{2}}{4 l_{B}^{2}}} g_{s}\left(x_{\mu}-y_{\mu}\right), \\
& \tilde{G}_{f}(x-y)=\frac{1}{4 \pi l_{B}^{2}} e^{-\frac{\left(x_{4}-y_{4}\right)^{2}+\left(x_{5}-y_{5}\right)^{2}}{4 l_{B}^{2}}} g_{f}\left(x_{\mu}-y_{\mu}\right)\left(1-i \Gamma_{4} \Gamma_{5}\right),
\end{aligned}
$$

where $g_{s}\left(x_{\mu}\right)$ and $g_{f}\left(x_{\mu}\right)$ are the ordinary scalar and fermion propagators in $(3+1)$ spacetime, given by

$$
\begin{aligned}
& g_{s}\left(x_{\mu}-y_{\mu}\right)=\int \frac{d p^{4}}{(2 \pi)^{4}} e^{-i p_{\mu}(x-y)^{\mu}} \frac{1}{p_{\mu}^{2}-m^{2}-|g B|} \\
& g_{f}\left(x_{\mu}-y_{\mu}\right)=\int \frac{d p^{4}}{(2 \pi)^{4}} e^{-i p_{\mu}(x-y)^{\mu}} \frac{p_{\mu} \Gamma^{\mu}+m}{p_{\mu}^{2}-m^{2}} .
\end{aligned}
$$


It is clear that the propagations along the extra dimension are indeed suppressed by the Gaussian function with the characteristic length $l_{B}$.

To consider in Feynman gauge $\xi=1$, we start from the differential equation

$$
\left[\left(D^{2}+m_{v}^{2}\right) \eta_{M N}-2 i g f_{M N}\right] G_{v}^{N P}(x, y)=i \delta_{M}^{P} \delta^{(6)}(x-y) .
$$

By applying a rotation matrix $\mathbf{P}$ with

$$
(\mathbf{P})_{M}^{N}=\left(\begin{array}{cccccc}
1 & 0 & 0 & 0 & 0 & 0 \\
0 & 1 & 0 & 0 & 0 & 0 \\
0 & 0 & 1 & 0 & 0 & 0 \\
0 & 0 & 0 & 1 & 0 & 0 \\
0 & 0 & 0 & 0 & \frac{1}{\sqrt{2}} & \frac{1}{\sqrt{2}} \\
0 & 0 & 0 & 0 & \frac{i}{\sqrt{2}} & \frac{-i}{\sqrt{2}}
\end{array}\right),
$$

Eq.(43) in the matrix form becomes

$$
\begin{aligned}
i 1 \delta^{(6)}(x-y) & =\mathbf{P}^{-1}\left[\left(D^{2}+m_{v}^{2}\right) \eta-2 i g \mathbf{f}\right] \mathbf{P P}^{-1} \mathbf{G P} \\
& =\left[D^{2}+m_{v}^{2}+2 g B \chi\right] \eta \cdot \hat{\mathbf{G}},
\end{aligned}
$$

where $\hat{\mathbf{G}} \equiv \mathbf{P}^{-1} \mathbf{G P}$ and $\chi=0,-1,1$ for $M=0 \sim 3,4,5$, respectively. It is clear that we can obtain the scalar propagator again with the matrix structure $g_{M N}$ and the replacement $m_{s}^{2} \rightarrow m_{v}^{2}+2 g B \chi$. The translational invariant part of the propagator for the vector field (up to a Schiwinger phase) is given by:

$$
\tilde{G}_{M N}(p)=\mathbf{P}_{M}^{P} \hat{G}_{P Q}(p)\left(\mathbf{P}^{-\mathbf{1}}\right)_{N}^{Q}=\mathbf{P}_{M}^{P}\left[\eta_{P Q} \times\left.\tilde{G}_{s}(p)\right|_{m_{s}^{2} \rightarrow m_{v}^{2}+2 g B \eta}\right]\left(\mathbf{P}^{-\mathbf{1}}\right)_{N}^{Q},
$$

where $G_{s}(p)$ is given in Eq. (32).

Some remarks are in order. First, we emphasize that we can take the limit $g \rightarrow 0$ but keep the combination $|g B|>>p_{\perp}^{2}$ to guarantee the use of the LLL. Secondly, as shown explicitly in Eqs. (37) and (38), when we consider a scattering amplitude, the momentum integral is effectively four-dimensional since the transverse momentum integral is factored out and becomes a Gaussian-type integral which is only a finite constant. This is still true even if we go beyond the LLL as we can see from Eqs. (34) and (35).

In summary, we have considered a simple way to localize scalar, fermion and abelian gauge fields on our 3-brane. The advantage of this consideration is that it is a simple and well-studied setup. We note that the remnant from the "magnetic field" gives effective mass 
terms for scalars even if we set the the bulk mass to zero, leading to an effective massive scalar theory in $(3+1)$ spacetime. On the other hand, if we start from a massless bulk fermion theory, the effective theory in $(3+1)$ spacetime will still be massless.

To localize a massive gauge field, an additional Stuckelberg field is needed to preserve the gauge invariance. The choice of this bulk mass term is crucial for us to avoid the "zeromode" problem. However, if we would like to reduce to an effective massless abelian gauge theory in $(3+1)$ spacetime, an imaginary mass could be considered, leading to a tachyonic

mode in the theory. This unavoidable tachyonic mode shall have interesting phenomenology and deserve further studies.

\section{ACKNOWLEDGMENTS}

We are grateful to Professor Roman Jackiw for many useful discussions and reading the manuscript. We would like to thank Yuhsin Tsai for helpful discussions. The work by S.H.H. is supported by the National Science Council of R.O.C. under Grant number: NSC98-2917I-564-122 and that by C.Q.G. is supported in part by the National Science Council of R.O.C. under Grant number: NSC-98-2112-M-007-008-MY3.

[1] For a review, see M. Shifman, Int. J. Mod. Phys. A 25, 199 (2010).

[2] V. A. Rubakov and M. E. Shaposhnikov, Phys. Lett. B 125, 136 (1983).

[3] G. A. Palma, Phys. Rev. D 73, 045023 (2006) arXiv:hep-th/0505170.

[4] R. F. Dashen, B. Hasslacher and A. Neveu, Phys. Rev. D 10, 4130 (1974).

[5] R. Jackiw and C. Rebbi, Phys. Rev. D 13, 3398 (1976).

[6] T. Haugset, J. Aa. Ruud and F. Ravndal, Phys. Scr. 47, 715 (1993).

[7] R. Jackiw, Phys. Rev. D 29, 2375 (1984) [Erratum-ibid. D 33, 2500 (1986)]

[8] R. Jackiw, Phys. Rev. D 57, 2635 (1998).

[9] For a review on the Stuckelberg formalism, see H. Ruegg and M. Ruiz-Altaba, Int. J. Mod. Phys. A 19, 3265 (2004).

[10] N. K. Nielsen and P. Olesen, Nucl. Phys. B 144, 376 (1978).

[11] A. Hashimoto and W. Taylor, Nucl. Phys. B 503, 193 (1997). 
[12] This dimensional reduction effect has been widely studied in $(3+1)$ and $(2+1)$ dimensions. See, for example, V. P. Gusynin, V. A. Miransky and I. A. Shovkovy, Nucl. Phys. B 563, 361 (1999). and reference therein.

[13] G. V. Dunne, R. Jackiw and C. A. Trugenberger, Phys. Rev. D 41, 661 (1990);

G. V. Dunne and R. Jackiw, Nucl. Phys. Proc. Suppl. 33C, 114 (1993);

R. Jackiw, Nucl. Phys. Proc. Suppl. 108, 30 (2002) [Phys. Part. Nucl. 33, S6 (2002)] [Lect. Notes Phys. 616, 294 (2003)].

[14] J. S. Schwinger, Phys. Rev. 82, 664 (1951).

[15] A. Chodos, K. Everding and D. A. Owen, Phys. Rev. D 42, 2881 (1990). 